\title{
UNKNOTTING SPHERES IN FIVE DIMENSIONS
}

\author{
BY E. C. ZEEMAN
}

Communicated by S. Eilenberg, December 26, 1959

Given a semi-linear embedding of $S^{2}$ in euclidean 5-space, we show that it is unknotted.

Join it up to a vertex $V$ in general position. If the cone $V S^{2}$ is nonsingular we are finished. Otherwise, for dimensional reasons, there are at most a finite number of singularities, where just two points of $S^{2}$ are collinear with $V$. Let's have $V$ away on one side, so that at each singularity we can call one point "near" and the other point "far." Now separate the near and far points by an equator $S^{1}$, so that all the near points lie in the northern hemisphere $A$, and all the far points lie in the southern hemisphere $B$.

Let $\hat{S}^{2}$ be the sphere $V S^{1} \cup B$. Then $\hat{S}^{2}$ is equivalent to $S^{2}$, because they differ by the boundary of the ball $V A$, whose interior does not meet them. But $\widehat{S}^{2}$ is unknotted because it bounds, and does not meet the interior of, the ball $V B$. Hence $S^{2}$ is unknotted.

REMARK 1. The argument generalizes to unknotting $S^{n}$ in $k$-space, $k>(3 / 2)(n+1)$.

REMARK 2. I suspect that $S^{3}$ knots in 6-space (the first unsolved case), because the near set can link the far set.

Gonville and Caius College,

Cambridge, England 